\title{
Variational methods for boundary value problems
}

\author{
B. TSANG \\ b.tsang@math.auckland.ac.nz \\ Department of Mathematics, University of Auckland, Auckland, New Zealand. \\ Department of Mathematics and Statistics, University of Canterbury, Christchurch, New Zealand. \\ S.W. TAYLOR \\ s.taylor@math.auckland.ac.nz \\ Department of Mathematics, University of Auckland, Auckland, New Zealand. \\ G.C. WAKE \\ g.wake@math.canterbury.ac.nz \\ Department of Mathematics and Statistics, University of Canterbury, Christchurch, New Zealand.
}

Dedicated to Dr. Alex McNabb on the occasion of his 70th birthday in January 2000 .

\begin{abstract}
The variational formulation of boundary value problems is valuable in providing remarkably easy computational algorithms as well as an alternative framework with which to prove existence results. Boundary conditions impose constraints which can be annoying from a computational point of view. The question is then posed: what is the most general boundary value problem which can be posed in variational form with the boundary conditions appearing naturally? Special cases of two-point problems in one-dimension and some higher dimensional problems are addressed. There is a deep connection with self-adjointness for the linear case. Further cases under which a Lagrangian may or may not exist are explained.
\end{abstract}

Keywords: natural boundary conditions, self-adjointness, Hamiltonian, Lagrangian.

\section{Introduction}

\subsection{Background}

Variational methods provide a conceptual and computational framework for boundary value problems (BVPs). In the paper we derive conditions which guarantee that a given BVP can be reformulated in variational form. This can be a way of proving existence for BVPs (Graham-Eagle and Wake [6]) and it also provides a scheme for determination of a computational algorithm as in Fradkin and Wake [5]. In the case of nonlinear eigenvalue problems, these can be a way of producing outcomes like parameter path-following as has been produced by other means by computer packages like Auto (Doedel [2]).

The question of what precise conditions need to be imposed on boundary value problems so that they can be written in variational form has been discussed spasmodically in the literature, see for example Anderson and Thompson [1], Fels [3], 
and Forray [4]. The question as to which boundary value problems have a variational formulation in which all of the boundary conditions appear naturally is largely still an open one and is the subject of this paper.

Boundary conditions appear naturally by the addition of suitable terms in the Hamiltonian (usually surface integrals on the boundary of the region of interest). It is usually difficult and sometimes impossible to determine these in a systematic way. Problems exist (like the two-point second order boundary value problem in one dimension with Dirichlet boundary conditions mentioned below) for which this is impossible. In these cases the boundary conditions need to be imposed on the space of functions on which the stationary values of the Hamiltonian is found, and are called "essential boundary conditions". This can be of nuisance value in the determination of the subspaces of functions on which the optimisation occurs. Thus it appears useful to be able to write the variational formulation with all of the boundary conditions appearing naturally. The more fundamental question as to whether there is even a Lagrangian is mentioned in Section 3.

\subsection{Conceptual framework}

If a variational formulation of our problem consists of the Hamiltonian functional $T: S \rightarrow \Re$, on the space of functions $S$ (suitably chosen), then $T$, under suitable conditions, has a stationary value on $S$. This gives rise to a boundary value problem. Our question is: how to choose $T$ so that it has the right boundary conditions which arises in the original problem. If a variational formulation has the boundary conditions imposed on $S$, they are said to be essential. However, if $T$ can be constructed so as to make the boundary conditions automatically satisfied on $S$, then they are said to be natural. The problem below shows, it is possible to have part, all, or none of the boundary conditions "natural" and the remaining therefore are "essential".

We initially thought that all self-adjoint problems (see Stakgold [9]) had a variational form (VF) in which the boundary conditions appear naturally. The simple problem,

$$
\begin{gathered}
y^{\prime \prime}+f(x) y+g(x)=0, \quad a<x<b, \\
y(a)=y(b)=0,
\end{gathered}
$$

is self-adjoint. However, the boundary conditions are essential and it can be considered as an example of Case 1 (vi) below. Hence not every self-adjoint boundary value problem has a VF in which the boundary conditions appear naturally. Nonetheless it appears that there is a substantial overlap between the class of boundary value problems which can be written in VF with natural boundary conditions and those that are self-adjoint. However, counter-examples like that in (1) provide evidence that there is not an exact correspondence. In Section 2, some of the conditions obtained are related to those of self-adjointness.

It appears impossible to completely characterise the class of BVPs which have $\mathrm{VF}$ with natural boundary conditions. In this paper we provide conditions on some 
classes of problems for which this is possible and give conditions under which this is true. The larger question is still open.

\section{Second order BVP in one-dimension}

Consider the second order ordinary differential equation

$$
y^{\prime \prime}+g\left(x, y, y^{\prime}\right)=0, \quad a<x<b,
$$

where $g\left(x, y, y^{\prime}\right)$ is a sufficiently smooth function. We are interested in solutions of (2) that satisfy the non-homogeneous linear boundary conditions

$$
\begin{aligned}
& m_{11} y(a)+m_{12} y(b)+n_{11} y^{\prime}(a)+n_{12} y^{\prime}(b)=k_{1}, \\
& m_{21} y(a)+m_{22} y(b)+n_{21} y^{\prime}(a)+n_{22} y^{\prime}(b)=k_{2} .
\end{aligned}
$$

We rewrite (3) in the vector form,

$$
M \mathbf{y}+N \mathbf{y}^{\prime}=\mathbf{k} .
$$

where $M, N$ are $2 \times 2$ matrices and $(M: N)$ has rank $=2$ to ensure $(3 a),(3 b)$ are independent. Suppose that the BVP consisting of (2) and (3) has a corresponding functional form,

$$
J[y]=\int_{a}^{b} f\left(x, y, y^{\prime}\right) d x+h\left(z_{1}, z_{2}\right),
$$

where $z_{1}=y(a)$ and $z_{2}=y(b)$ and $h\left(z_{1}, z_{2}\right) \in C^{2}$.

The boundary value problem is related to the corresponding functional form by the Euler-Langrange equation. By setting the first variation $\delta J$ of $J$ to zero,

$$
\begin{aligned}
\delta J & =\int_{a}^{b}\left(\frac{\partial f}{\partial y} \delta y+\frac{\partial f}{\partial y^{\prime}} \delta y^{\prime}\right) d x+\frac{\partial h}{\partial z_{1}} \delta y(a)+\frac{\partial h}{\partial z_{2}} \delta y(b), \\
& =\left[\frac{\partial f}{\partial y^{\prime}} \delta y\right]_{a}^{b}+\int_{a}^{b}\left[\frac{\partial f}{\partial y}-\frac{d}{d x}\left(\frac{\partial f}{\partial y^{\prime}}\right)\right] \delta y d x+\frac{\partial h}{\partial z_{1}} \delta y(a)+\frac{\partial h}{\partial z_{2}} \delta y(b), \\
& =0,
\end{aligned}
$$

we find that the following conditions must be satisfied by the boundary terms $h$ in the functional $J$ and the boundary conditions:

$$
\frac{\partial h}{\partial z_{1}}=\left.\frac{\partial f}{\partial y^{\prime}}\right|_{x=a} \quad \text { and } \quad \frac{\partial h}{\partial z_{2}}=-\left.\frac{\partial f}{\partial y^{\prime}}\right|_{x=b} .
$$

Let $q_{1}\left(z_{1}, z_{2}\right)=\left.\frac{\partial f}{\partial y^{\prime}}\right|_{x=a}$ and $q_{2}\left(z_{1}, z_{2}\right)=-\left.\frac{\partial f}{\partial y^{\prime}}\right|_{x=b}$. By partially integrating $q_{1}$ and $q_{2}$ with respect to $z_{1}$ and $z_{2}$ respectively, we can find the boundary functional terms $h\left(z_{1}, z_{2}\right)$, provided that a certain exactness or integrability condition is satisfied. That is

$$
\frac{\partial^{2} h}{\partial z_{1} \partial z_{2}}=\frac{\partial^{2} h}{\partial z_{2} \partial z_{1}}
$$

as we require that $h\left(z_{1}, z_{2}\right) \in C^{2}$. 


\subsection{Case 1: $g$ independent of $y^{\prime}$}

For simplicity and without loss of generality, we let $[a, b]=[0,1]$,

$$
\begin{gathered}
y^{\prime \prime}+g(x, y)=0, \quad 0<x<1, \\
M \mathbf{y}+N \mathbf{y}^{\prime}=\mathbf{k} .
\end{gathered}
$$

The corresponding variational formulation is

$$
J[y]=\int_{0}^{1}\left[\frac{\left(y^{\prime}\right)^{2}}{2}-G(x, y)\right] d x+h\left(z_{1}, z_{2}\right),
$$

where $G$ is a partial primitive of $g$ with respect to $y$, i.e. $\frac{\partial G}{\partial y}=g$. The first variation of $J$ is

$$
\delta J=\left[y^{\prime} \delta y\right]_{0}^{1}-\int_{0}^{1}\left[\frac{\partial G}{\partial y}+y^{\prime \prime}\right] \delta y d x+\frac{\partial h}{\partial z_{1}} \delta y(0)+\frac{\partial h}{\partial z_{2}} \delta y(1) .
$$

There are several types boundary functional terms $h\left(z_{1}, z_{2}\right)$ formed in the variational formulation which are shown as the following. Let $[\tilde{N}: \tilde{M}]$ be the row reduced echelon form of the augmented matrix $[N: M]$. There are only six possible cases and these are analysed below. Cases $(i)-(i v)$ are BVPs with at least one natural boundary condition in their variational forms. Cases $(v)$ and $(v i)$ are impossible to write in this form with any natural boundary condition, that is, they have to be imposed as essential boundary conditions.

\section{(i) Mixed boundary conditions I}

$$
[\tilde{N}: \tilde{M}] \cong\left[\begin{array}{llll}
1 & 0 & \alpha_{1} & \alpha_{2} \\
0 & 1 & \alpha_{3} & \alpha_{4}
\end{array}\right]
$$

The boundary conditions are

$$
\begin{aligned}
& \alpha_{1} y(0)+\alpha_{2} y(1)+y^{\prime}(0)=k_{1}, \\
& \alpha_{3} y(0)+\alpha_{4} y(1)+y^{\prime}(1)=k_{2},
\end{aligned}
$$

which are Neumann boundary conditions if all the $\alpha$ 's are zero. Then putting $\delta J=0$ in equation (10), we see that the boundary terms yield the conditions,

$$
\begin{aligned}
& \frac{\partial h}{\partial z_{1}}=k_{1}-\alpha_{1} z_{1}-\alpha_{2} z_{2}, \\
& \frac{\partial h}{\partial z_{2}}=-\left(k_{2}-\alpha_{3} z_{1}-\alpha_{4} z_{2}\right) .
\end{aligned}
$$

Then integrating equations (11) and (12) partially to get $h$, we find,

$$
h\left(z_{1}, z_{2}\right)=-\frac{1}{2} \alpha_{1} z_{1}^{2}-\alpha_{2} z_{1} z_{2}+\frac{1}{2} \alpha_{4} z_{2}^{2}+k_{1} z_{1}-k_{2} z_{2} .
$$


provided that $\alpha_{3}=-\alpha_{2}$, which follows from the exactness condition (7).

(ii) Mixed boundary conditions II

$$
[\tilde{N}: \tilde{M}] \cong\left[\begin{array}{cccc}
1 & \alpha_{1} & 0 & \alpha_{2} \\
0 & 0 & 1 & \alpha_{3}
\end{array}\right]
$$

The boundary conditions are

$$
\begin{aligned}
\alpha_{2} y(1)+y^{\prime}(0)+\alpha_{1} y^{\prime}(1) & =k_{1}, \\
y(0)+\alpha_{3} y(1) & =k_{2} .
\end{aligned}
$$

In this case, we find that

$$
h=\frac{1}{2} \alpha_{2} \alpha_{3} z_{2}^{2}-\alpha_{3} k_{1} z_{2},
$$

provided that $\alpha_{1} \alpha_{3}=1$ which again follows from the exactness condition (7). Note that in this case only the second boundary condition is essential in that it must be imposed on the space of functions considered.

(iii) Mixed boundary conditions III

$$
[\tilde{N}: \tilde{M}] \cong\left[\begin{array}{cccc}
1 & \alpha_{1} & \alpha_{2} & 0 \\
0 & 0 & 0 & 1
\end{array}\right]
$$

The boundary conditions are

$$
\begin{aligned}
\alpha_{2} y(0)+y^{\prime}(0)+\alpha_{1} y^{\prime}(1) & =k_{1}, \\
y(1) & =k_{2},
\end{aligned}
$$

then $h$ is

$$
h=-\frac{\alpha_{2}}{2} z_{1}^{2}+k_{1} z_{1}
$$

provided $\alpha_{1}=0$ from the exactness condition (7). In this case only the second boundary condition is essential.

(iv) Mixed boundary conditions IV

$$
[\tilde{N}: \tilde{M}] \cong\left[\begin{array}{llll}
0 & 1 & 0 & \alpha_{1} \\
0 & 0 & 1 & \alpha_{2}
\end{array}\right]
$$

The boundary conditions are

$$
\begin{aligned}
\alpha_{1} y(1)+y^{\prime}(1) & =k_{1}, \\
y(0)+\alpha_{2} y(1) & =k_{2},
\end{aligned}
$$

then $h$ is

$$
h=\frac{\alpha_{1}}{2} z_{2}^{2}-k_{1} z_{2}
$$


provided that $\alpha_{2}=0$ from the exactness condition (7). In this case only the second boundary condition is essential.

\section{(v) Mixed boundary conditions $\mathbf{V}$}

$$
[\tilde{N}: \tilde{M}] \cong\left[\begin{array}{cccc}
0 & 1 & \alpha_{1} & 0 \\
0 & 0 & 0 & 1
\end{array}\right]
$$

The boundary conditions are

$$
\begin{aligned}
\alpha_{1} y(0)+y^{\prime}(1) & =k_{1}, \\
y(1) & =k_{2} .
\end{aligned}
$$

By calculating the first variation of $J$ and accepting that the second boundary condition is inevitably an essential boundary condition, we can then find the condition

$$
\frac{d h}{d z_{1}}=y^{\prime}(0) .
$$

It is impossible to get a solution for the boundary functional terms $h$. Hence neither boundary condition is natural in this case.

\section{(vi) Unmixed boundary conditions}

$$
[\tilde{N}: \tilde{M}] \cong\left[\begin{array}{llll}
0 & 0 & 1 & 0 \\
0 & 0 & 0 & 1
\end{array}\right]
$$

With the boundary conditions,

$$
\begin{aligned}
& y(0)=k_{1}, \\
& y(1)=k_{2} .
\end{aligned}
$$

This is the Dirichlet problem and in this case, it is sufficient to take $h \equiv 0$. Note that both boundary conditions are essential in this case.

All cases for which $h$ exists satisfy the condition $\tilde{m}_{11} \tilde{n}_{21}-\tilde{m}_{21} \tilde{n}_{11}=\tilde{m}_{12} \tilde{n}_{22}-$ $\tilde{m}_{22} \tilde{n}_{12}$ which is equivalent to the condition $m_{11} n_{21}-m_{21} n_{11}=m_{12} n_{22}-m_{22} n_{12}$ because the equation is invariant for elementary row operations on $[N, M]$. Hence we obtain the following theorem.

Theorem A boundary value problem of the form

$$
\begin{gathered}
y^{\prime \prime}+g(x, y)=0, \quad a<x<b, \\
M \mathbf{y}+N \mathbf{y}^{\prime}=\mathbf{k},
\end{gathered}
$$

with non-zero matrix $N$ has the corresponding variational formulation with functional of the form

$$
J[y]=\int_{a}^{b}\left(\frac{\left(y^{\prime}\right)^{2}}{2}-G(x, y)\right) d x+h\left(z_{1}, z_{2}\right),
$$


if and only if $m_{11} n_{21}-m_{21} n_{11}=m_{12} n_{22}-m_{22} n_{12}$.

The conditions above on $M, N$ are just the conditions that the boundary conditions are self-adjoint. In the cases $(i)-(i v)$ the extra conditions on the $\alpha_{i}$ 's are just those that make the problems self-adjoint.

\subsection{Case 2: $g$ dependent on $y^{\prime}$}

Here a more basic question arises: what is the basic form of the Lagrangian $f$ ? It is clear that not all boundary value problems have a Lagrangian. ( e.g. $y^{\prime}=g(x, y)$ a first order differential equation, does not have a VF. ) This question is discussed generally in Olver [8].

Suppose there is a second order ordinary differential equation,

$$
y^{\prime \prime}+g\left(x, y, y^{\prime}\right)=0, \quad x \in[a, b]
$$

where $g\left(x, y, y^{\prime}\right)$ is a smooth function. We wish to see if a variational formulation exists in the form,

$$
J[y]=\int_{a}^{b} f\left(x, y, y^{\prime}\right) d x,
$$

which should be optimised by the solution of the ODE. We wish to investigate if the corresponding first variation of the functional $J[y]$ could produce a functionally independent but compatible differential equation for $y$ so that any solution to the new equation is also a solution of the in the original boundary value problem. The Euler-Lagrange equation is:

$$
\frac{\partial f}{\partial y}-\frac{d}{d x}\left(\frac{\partial f}{\partial y^{\prime}}\right)=\frac{\partial f}{\partial y}-\frac{\partial^{2} f}{\partial x \partial y^{\prime}}-\frac{\partial^{2} f}{\partial y \partial y^{\prime}} y^{\prime}-\frac{\partial^{2} f}{\partial\left(y^{\prime}\right)^{2}} y^{\prime \prime}=0 .
$$

Put $p=y^{\prime}$ and $y^{\prime \prime}=-g\left(x, y, y^{\prime}\right)$ for simplification. Then $f$ must satisfy

$$
\frac{\partial^{2} f}{\partial x \partial p}+p \frac{\partial^{2} f}{\partial y \partial p}-g \frac{\partial^{2} f}{\partial p^{2}}=\frac{\partial f}{\partial y} .
$$

The equation is most easily solved if we first differentiate it with respect to $p$

$$
\frac{\partial^{3} f}{\partial x \partial p^{2}}+\frac{\partial^{2} f}{\partial y \partial p}+p \frac{\partial^{3} f}{\partial y \partial p^{2}}-\frac{\partial g}{\partial p} \frac{\partial^{2} f}{\partial p^{2}}-g \frac{\partial^{3} f}{\partial p^{3}}=\frac{\partial^{2} f}{\partial y \partial p} .
$$

Now put

$$
\frac{\partial^{2} f}{\partial p^{2}}=z
$$


Then we get the first order partial differential equation

$$
\frac{\partial z}{\partial x}+p \frac{\partial z}{\partial y}-g \frac{\partial z}{\partial p}=z \frac{\partial g}{\partial p} .
$$

Consider the characteristic equations,

$$
\begin{aligned}
& \frac{d x}{d s}=1 \\
& \frac{d y}{d s}=p \\
& \frac{d p}{d s}=-g \\
& \frac{d z}{d s}=z \frac{\partial g}{\partial p}
\end{aligned}
$$

where $s$ is a characteristic variable (see [7] for detail). We have

$$
z=e^{\int \frac{\partial g}{\partial p} d s}
$$

and for $g$ is "nice enough", $\int \frac{\partial g}{\partial p} d s$ will always exist. Then, the Lagrangian $f$ can be found by integrating $z$ twice with respect to $p$.

Explicit answers for $f$ are provided for the following cases.

(i) $g=Q(p) R(x, y)$

If $z$ depends only on $p$, substitute $g$ into (16) to get $z=\frac{1}{Q(p)}$. So, the Lagrangian $f$ will be,

$$
f=\int_{0}^{p} \int_{0}^{u} \frac{1}{Q(s)} d s d u+A(x, y) p+B(x, y) .
$$

Putting $f$ into the Euler-Lagrange equation shows that,

$$
R(x, y)=\frac{\partial A}{\partial x}-\frac{\partial B}{\partial y} .
$$

If we choose $A=0, B=-\int_{0}^{y} R(x, u) d u$ then the Lagrangian is

$$
f=\int_{0}^{p} \int_{0}^{u} \frac{1}{Q(s)} d s d u-\int_{0}^{y} R(x, u) d u .
$$

(ii) $g=R(x) p+Q(x, y)$

From equation (16) we get $z=e^{\int_{0}^{x} R(s) d s}$, and the Lagrangian is

$$
f=\frac{p^{2}}{2} e^{\int_{0}^{x} R(s) d s}+A(x, y) p+B(x, y) .
$$


Then putting (18) into the Euler-Lagrange equation we obtain

$$
Q(x, y)=\left(\frac{\partial A}{\partial x}-\frac{\partial B}{\partial y}\right) e^{-\int_{0}^{x} R(s) d s},
$$

and we obtain $A, B$ as in $(i)$.

(iii) $g=R(x) p+Q(x) y+P(x)$

This is a particular case of $(19)$ with $Q(x, y)$ linear in $y$,

$$
Q(x) y+P(x)=\left(\frac{\partial A}{\partial x}-\frac{\partial B}{\partial y}\right) e^{-\int_{0}^{x} R(s) d s} .
$$

(a) We can choose $A$ and $B$ as,

$$
A(x, y)=0,
$$

and

$$
\frac{\partial B}{\partial y}=-(Q(x) y+P(x)) e^{\int_{0}^{x} R(s) d s} .
$$

In this case, the Lagrangian will be

$$
f=\frac{p^{2}}{2} e^{\int_{0}^{x} R(s) d s}-\left(\frac{y^{2}}{2} Q(x)+P(x) y\right) e^{\int_{0}^{x} R(s) d s} .
$$

(b) There are other choices for $A$ and $B$. With

$$
\begin{gathered}
A(x, y)=y \int_{0}^{x} Q(s) d s e^{\int_{0}^{x} R(s) d s} \\
B(x, y)=-h(x) y e^{\int_{0}^{x} R(s) d s}+\frac{1}{2} y^{2} R(x) \int_{0}^{x} Q(s) d s e^{\int_{0}^{x} R(s) d s},
\end{gathered}
$$

we get the Lagrangian

$$
f=\left(\frac{p^{2}}{2}+y p \int_{0}^{x} Q(s) d s-h(x) y+\frac{1}{2} y^{2} R(x) \int_{0}^{x} Q(s) d s\right) e^{\int_{0}^{x} R(s) d s} .
$$

Thus there are only these few cases of equation (13) for which a Lagrangian can be constructed for the VF of the boundary value problem. In these cases only we would then find conditions on the $\alpha_{k}$ 's for which the boundary conditions could be natural, using the compatibility conditions in equation (7). The details are not given here. 


\section{Extensions}

In this section, we will look at the usage of VF in partial differential equations and the relation with self-adjointness. Again the challenge is to find the Lagrangian first. A parallel work can be found in Olver [8], which shows a homogenous system of linear differential equations has a corresponding functional form if and only if the differential operator is self-adjoint.

\subsection{Higher dimensional systems}

Consider a second order partial differential equation of the form,

$$
q_{1}(\mathbf{x}, y)\left(\sum_{i j} a_{i j}(\mathbf{x}) \frac{\partial^{2} y}{\partial x_{i} \partial x_{j}}+\sum_{j} b_{j}(\mathbf{x}) \frac{\partial y}{\partial x_{j}}+c(\mathbf{x}) y\right)=0, \quad \mathbf{x} \in \Omega \subseteq \Re^{n},
$$

where $q_{1}(\mathbf{x}, y)$ is an arbitrary multiple function which may arise when the partial differential equation is evaluated from the Euler-Lagrange equations. Then the corresponding Lagrangian has the form,

$$
f=\sum_{i j} a_{i j}(\mathbf{x}) q_{1}(\mathbf{x}, y) \frac{\partial y}{\partial x_{i}} \frac{\partial y}{\partial x_{j}}+q(\mathbf{x}, y),
$$

where $q(\mathbf{x}, y)=q_{1}(\mathbf{x}, y) q_{2}(\mathbf{x}, y)$ and $q_{2}(\mathbf{x}, y)$ is an arbitrary function. By putting the Lagrangian $f$ into the Euler-Lagrange equations, we get,

$$
q_{1}=q_{1}(\mathbf{x}) \quad \text { and } \quad q_{2}(\mathbf{x}, y)=-\frac{1}{2} c(\mathbf{x}) y^{2} .
$$

Then, to solve for $q_{1}(\mathbf{x})$, we need to solve the equations,

$$
\sum_{i} \frac{\partial}{\partial x_{i}}\left(a_{i j}(\mathbf{x}) q_{1}(\mathbf{x})\right)=q_{1}(\mathbf{x}) b_{j}(\mathbf{x}), \quad j=1, \ldots, n .
$$

If $q_{1}(\mathbf{x})$ is a constant, the equations (22) become

$$
\sum_{i} \frac{\partial}{\partial x_{i}}\left(a_{i j}(\mathbf{x})\right)=b_{j}(\mathbf{x}) .
$$

This means the partial differential equations are self-adjoint and the funtional form is

$$
J[y]=\int_{\Omega}\left(\sum_{i j} a_{i j}(\mathbf{x}) \frac{\partial y}{\partial x_{i}} \frac{\partial y}{\partial x_{j}}-c(\mathbf{x}) y^{2}\right) d \mathbf{x} .
$$


However, if $q_{1}(\mathbf{x})$ is not a constant, the equations (22) are

$$
\sum_{i}\left(a_{i j}(\mathbf{x}) \frac{\partial q_{1}}{\partial x_{i}}+q_{1}(\mathbf{x}) \frac{\partial a_{i j}}{\partial x_{i}}\right)=q_{1}(\mathbf{x}) b_{j}(\mathbf{x}) .
$$

Since $A=\left(a_{i j}\right)$ is symmetric, $a_{i j}=a_{j i}$, so that

$$
\sum_{i} \frac{1}{q_{1}} \frac{\partial q_{1}}{\partial x_{i}} a_{j i}=b_{j}-\sum_{i} \frac{\partial a_{i j}}{\partial x_{i}} .
$$

This can be thought of as the jth row of the system of equations,

$$
A\left(\begin{array}{c}
\frac{1}{q_{1}} \frac{\partial q_{1}}{\partial x_{1}} \\
\frac{1}{q_{1}} \frac{\partial q_{1}}{\partial x_{2}} \\
\vdots \\
\frac{1}{q_{1}} \frac{\partial q_{1}}{\partial x_{n}}
\end{array}\right)=\left[\begin{array}{c}
b_{1}-\sum_{i} \frac{\partial a_{i 1}}{\partial x_{i}} \\
b_{2}-\sum_{i} \frac{\partial x_{i 2}}{\partial x_{i}} \\
\vdots \\
b_{n}-\sum_{i} \frac{\partial a_{i n}}{\partial x_{i}}
\end{array}\right]
$$

or

$$
A \nabla\left(\ln q_{1}\right)=b-\nabla \cdot A .
$$

If the $n$ equations for $q_{1}$ are compatible with each other and satisfy the system, then $q_{1}$ can be found. The variational formulation for the linear non-self-adjoint partial differential equation also exists.

\subsection{Example}

If $q_{1}$ is a constant equal to unity, we can have a second order linear self-adjoint partial differential equation,

$$
a\left(x_{1}, x_{2}\right) \frac{\partial^{2} y}{\partial x_{1}^{2}}+2 b\left(x_{1}, x_{2}\right) \frac{\partial^{2} y}{\partial x_{1} \partial x_{2}}+c\left(x_{1}, x_{2}\right) \frac{\partial^{2} y}{\partial x_{2}^{2}}+g\left(x_{1}, x_{2}, y, \frac{\partial y}{\partial x_{1}}, \frac{\partial y}{\partial x_{2}}\right)=0 .
$$

Comparing equation (23) with the Euler-Lagrange equation,

$$
\frac{d}{d x_{1}}\left(\frac{\partial f}{\partial p_{1}}\right)+\frac{d}{d x_{2}}\left(\frac{\partial f}{\partial p_{2}}\right)=\frac{\partial f}{\partial y}
$$

where $p_{i}=\frac{\partial y}{\partial x_{i}}$ and the ordinary differential operator $\frac{d}{d x_{i}}$ is considered as a special kind of partial differential operator which takes $x_{j}, j \neq i$, as constant. So

$$
\begin{aligned}
\frac{\partial^{2} f}{\partial p_{1}^{2}} \frac{\partial^{2} y}{\partial x_{1}^{2}}+2 \frac{\partial^{2} f}{\partial p_{1} \partial p_{2}} \frac{\partial^{2} y}{\partial x_{1} \partial x_{2}}+\frac{\partial^{2} f}{\partial p_{2}^{2}} \frac{\partial^{2} y}{\partial x_{2}^{2}} & +\frac{\partial^{2} f}{\partial y \partial p_{1}} \frac{\partial y}{\partial x_{1}}+\frac{\partial^{2} f}{\partial y \partial p_{2}} \frac{\partial y}{\partial x_{2}} \\
& +\frac{\partial^{2} f}{\partial x_{1} \partial p_{1}}+\frac{\partial^{2} f}{\partial x_{2} \partial p_{2}}=\frac{\partial f}{\partial y} .
\end{aligned}
$$


We can find the following conditions,

$$
\frac{\partial^{2} f}{\partial p_{1}^{2}}=a\left(x_{1}, x_{2}\right), \quad \frac{\partial^{2} f}{\partial p_{1} \partial p_{2}}=b\left(x_{1}, x_{2}\right), \quad \frac{\partial^{2} f}{\partial p_{2}^{2}}=c\left(x_{1}, x_{2}\right) .
$$

Then the Lagrangian $f$ will have the quadratic form,

$$
f=\frac{1}{2} A\left(x_{1}, x_{2}\right) p_{1}^{2}+B\left(x_{1}, x_{2}\right) p_{1} p_{2}+\frac{1}{2} C\left(x_{1}, x_{2}\right) p_{2}^{2}+D\left(x_{1}, x_{2}, y\right) .
$$

By putting the Lagrangian $f$ into the Euler-Lagrange equation, we will find that $D\left(x_{1}, x_{2}, y\right)=D_{1}\left(x_{1}, x_{2}\right) y+D_{2}\left(x_{1}, x_{2}\right)$ and

$$
f=\frac{1}{2} A\left(x_{1}, x_{2}\right) p_{1}^{2}+B\left(x_{1}, x_{2}\right) p_{1} p_{2}+\frac{1}{2} C\left(x_{1}, x_{2}\right) p_{2}^{2}+D_{1}\left(x_{1}, x_{2}\right) y+D_{2}\left(x_{1}, x_{2}\right) .
$$

For Laplace's equation, $a\left(x_{1}, x_{2}\right)=c\left(x_{1}, x_{2}\right)=1$ and $b\left(x_{1}, x_{2}\right)=0$ and the corresponding functional form is

$$
f=\frac{1}{2}\left(p_{1}^{2}+p_{2}^{2}\right)+D_{1}\left(x_{1}, x_{2}\right) y+D_{2}\left(x_{1}, x_{2}\right)
$$

with $D_{1}=0$, otherwise, it becomes Poisson's equation.

Acknowledgement: We thank Dr. Bruce van-Brunt for helpful suggestions.

\section{References}

1. Anderson, I. and Thompson, G., The Inverse Problem of the Calculus of Variations for Ordinary Differential Equations, Mem. Amer. Math. Soc., 98(no.473), (1992). MR 92k:58070.

2. Doedol, E., AUTO: Software for continuation and bifurcation problems in ordinary differential equations, California Institute of Technology, U.S.A., (1986)

3. Fels, M.E., The Inverse Problem of the Calculus of Variations for Scalar Fourth-order Ordinary Differential Equations, American Mathematical Society, Vol.348 Number 12, Dec. (1996).

4. Forray, M.J., Variational Calculus in Science and Engineering, New York, McGraw-Hill, (1968).

5. Fradkin, L.Ju. and Wake, G.C., The Critical Explosion Parameter in the Theory of Thermal Ignition, J. Inst. Maths Applics 20, 471-484, (1977).

6. Graham-Eagle, J.G. and Wake, G.C., Existence of solutions to a nonlinear boundary value problem by variational means, J. Math. Anal. Appl. Vol 92, 581-598, (1983).

7. Kevorkian, J., Partial Differential Equations, Analytical Solution Techniques, Pacific Grove, Calif. : Wadsworth \& Brooks/Cole Advanced Books \& Software, (1990).

8. Olver, P.J., Applications of Lie Group to Differential Equations, Springer-Verlag, GTM 107, (1986).

9. Stakgold, I., Green's Functions and Boundary Value Problems, New York, Wiley, (1979). 


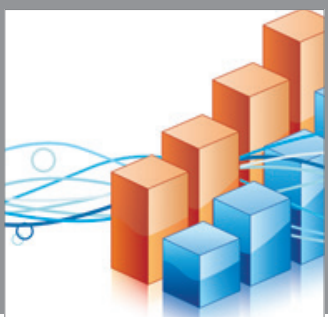

Advances in

Operations Research

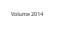

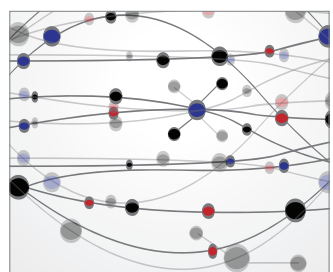

\section{The Scientific} World Journal
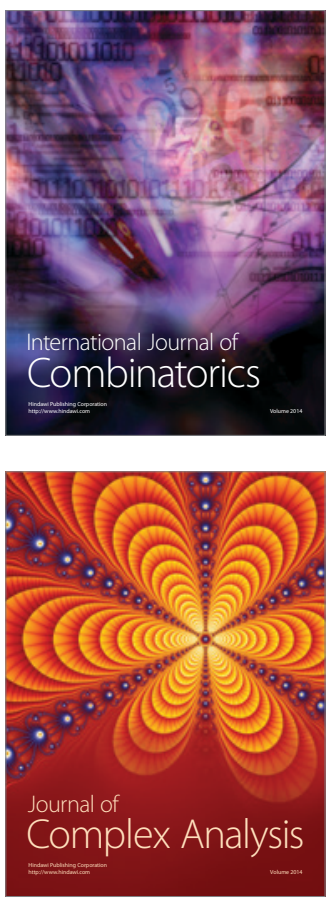

International Journal of

Mathematics and

Mathematical

Sciences
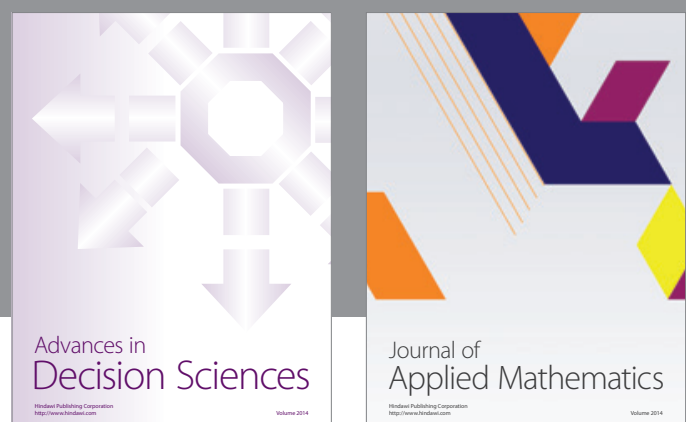

Journal of

Applied Mathematics
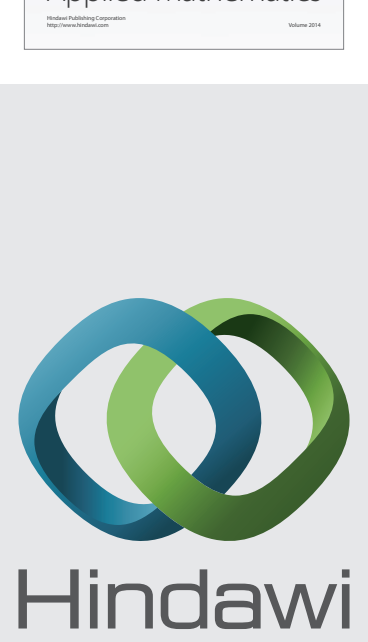

Submit your manuscripts at http://www.hindawi.com
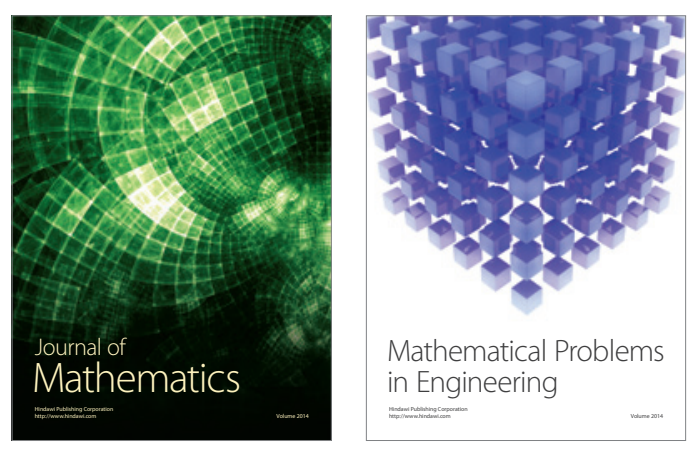

Mathematical Problems in Engineering
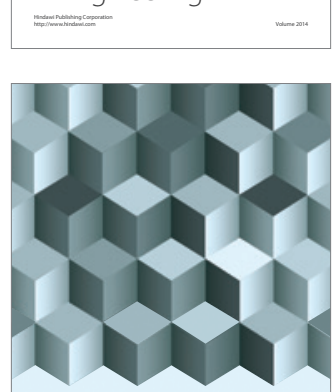

Journal of

Function Spaces
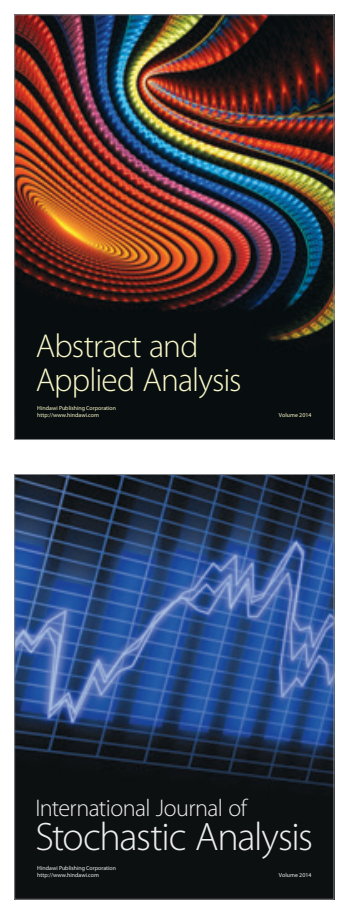

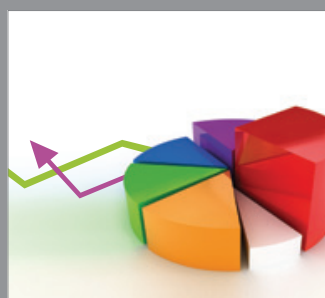

ournal of

Probability and Statistics

Promensencen
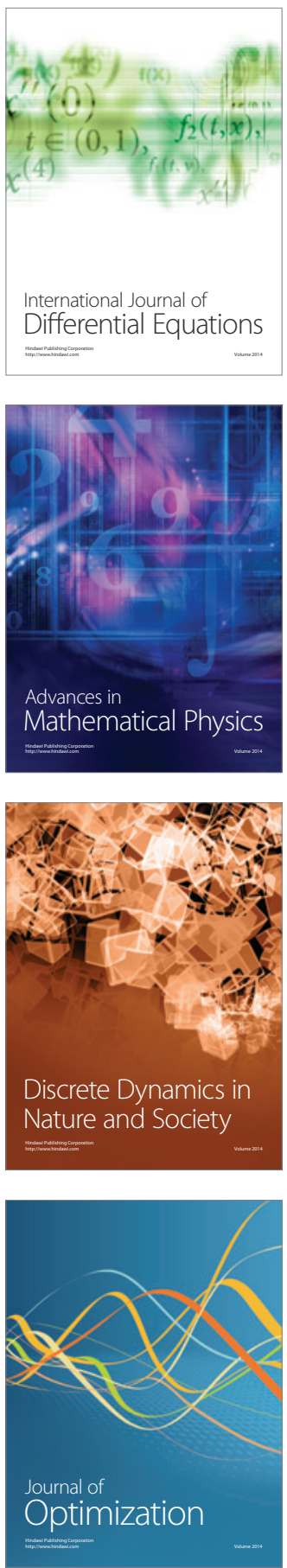